\title{
Design of Horizontal Tube Chain Conveyor Scraper Pitch
}

\author{
Liu Weili ${ }^{1}$, Yao Yanping ${ }^{2}$, Guo Xiaopeng ${ }^{3}$, Zhao Jianfei ${ }^{2}$ \\ ${ }^{1}$ Taiyuan Institute of China Coal Technology\&Engineering Corp, Taiyuan 030006, Shanxi, China \\ ${ }^{2}$ School of Mechanical Engineering, Taiyuan University of Science and Technology, Taiyuan 030024, China \\ ${ }^{3}$ Shanxi Taigang Stainless Steel Co.,Ltd., Taiyuan 030006, Shanxi, China
}

\begin{abstract}
Tube chain conveyor is a new type of energy saving and high efficient continuous conveying equipment with flexible traction components. Due to the lack of theory research, in the domestic TCC compared with the foreign one there are some shortcomings including high energy consumption, small size, small applicable scope, etc. According to the limit equilibrium conditions of bulk material, we will research the scraper's static force acts on the material and the force's transmission area of scraper to get the tube chain conveyor scraper pitch's limit calculation formula. It is of great significance to promote the development of domestic tube chain conveyor.
\end{abstract}

Keywords - Horizontal tube chain conveyor, scraper pitch, scraper forces, pipe chain conveyor structure, equilibrium conditions of bulk

\section{Introduction}

Pipe chain conveyor is a kind of continuous transport machinery with flexible traction components, with simple structure, easy to install, enclosed conveying, low energy consumption, low noise, flexible transport line layout, wide range of application and other characteristics.

DOI: $10.18421 /$ TEM94-12

https://doi.org/10.18421/TEM94-12

Corresponding author: Zhao Jianfei, School of Mechanical Engineering, Taiyuan University of Science and Technology, Taiyuan 030024, China.

Email: tykjdxzif@163.com

Received: 25 April 2020.

Revised: 17 August 2020.

Accepted: 25 August 2020.

Published: 27 November 2020.

(cc)BY-NC-ND (C) 2020 Liu Weili at al; published by UIKTEN. This work is licensed under the Creative Commons Attribution-NonCommercial-NoDerivs 4.0 License.

The article is published with Open Access at www.temjournal.com
In foreign countries, it is widely used in medicine, chemical industry, agriculture, coal, electric power, etc. Domestically, the research on pipe chain conveyor is relatively lagging. After nearly ten years of research, its performance has been improved to some extent, but compared with foreign countries, it has small scale, high energy consumption and small application range.

Although the tube chain conveyor has a simple structure, the design theory has its own unique features. The mechanical model directly determines the performance of the product [1]. The use of domestic pipe chain conveyors is currently mainly for the transportation of chemical raw materials such as PAT and PET. With the improvement of theoretical research and the improvement of manufacturing level, pipe chain conveyors will be adopted by more industries as a new type of conveying equipment [2].

\section{Pipe Chain Conveyor Structure and Principle}

\subsection{Pipe Chain Conveyor Structure}

Pipe chain conveyor is mainly composed of tension device 1 , the reversing device 2 , the feeding device 3 , the chain 4 , the circular scraper 5 , the circular trough 6 , the manhole 7 , the driving device 8 , the unloading device 9, and the like. As shown in Figure 1.

\subsection{Pipe Chain Conveyor Principle}

The space formed by any two adjacent scraper plates and circular tube feeding trough of the pipe chain conveyor is equivalent to an independent container. During work, materials that enter the feeding trough from feeding port form a material layer with an average height of $h$ between adjacent scraper plates. As shown in Figure 2., the velocity of the material layer changes from the initial velocity falling from the feeding port to the same velocity $\mathrm{v}$ as the scraper chain, thus forming a stable material flow. The material flow can only be pulled by the 
scraper chain and overcome the friction resistance with the trough wall to continuously forward transport until the unloading port is removed. One side of the trough is the bearing branch and the other side is the return branch.

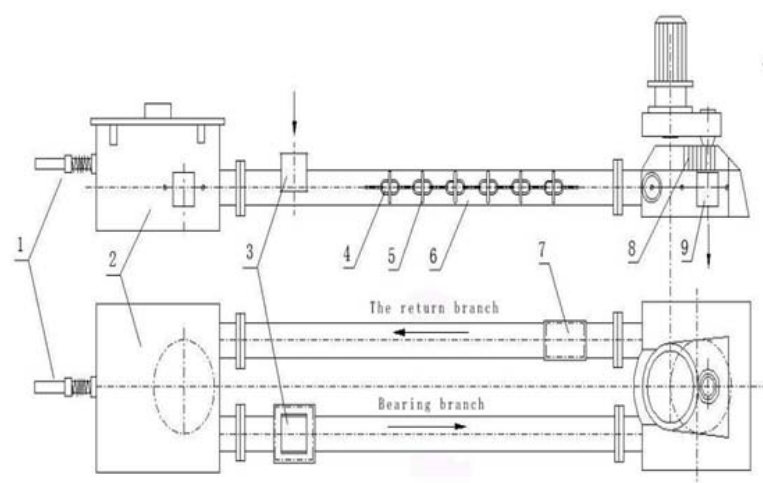

Figure 1. Horizontal tube scraper conveyor structure

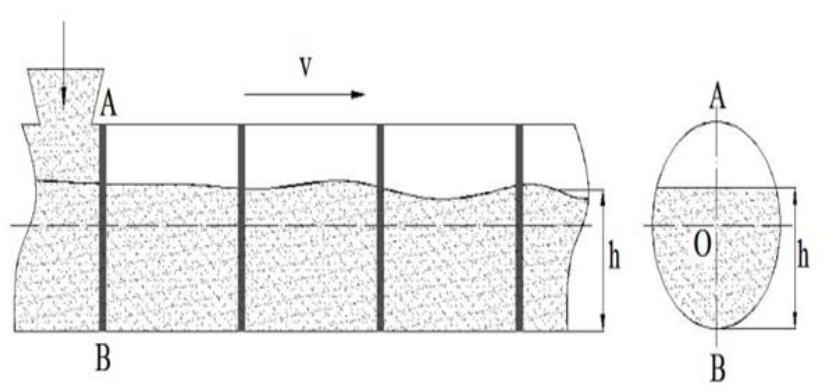

Figure 2. Horizontal conveying schematic diagram

\section{Mechanical Analysis of Pipe Chain Conveyor}

By virtue of the retaining wall theory of granular mechanics and soil mechanics, the material is subjected to passive pressure under the push of the scraper. When the push of the scraper makes the material reach the ultimate equilibrium state, the interaction force between the scraper and the material also reaches the ultimate state [3].

The material adjacent to the scraper $\mathrm{AB}$ and between, is taken as the research unit along the transport direction. The gap between the scraper and the trough is ignored. The material with a height of $h$ in the trough is transported forward under the push of the scraper $\mathrm{AB}$.

The internal diameter of the groove and the diameter of the scraper are set to be $\mathrm{D}$. The force exerted by the scraper on the material is $F_{x}$. It is distributed on the contact surface of the scraper and the granular material. The contact surface area $S_{0}$ between material and scraper and the stress $\sigma_{X}$ on the contact surface are as follows [4]:

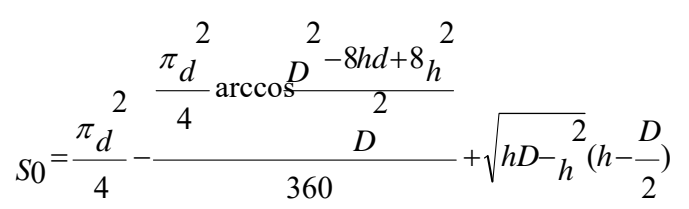

$$
\sigma_{X}=\frac{F_{X}}{S_{0}}
$$

According to the limit equilibrium condition of bulk material, when the value of $F_{x}$ reaches a certain limit, the shear stress generated in the material will exceed its shear strength, and the slip plane AC will be generated in the material to break the material, as shown in Figure 3. The slip surface $\mathrm{AC}$ is a curved surface. According to the analysis of the bulk mechanics, the plane can be used instead of the AC surface to study. The results obtained are also very accurate for practical applications [5]. At this time, since the scraper chain has a certain force, and the material is broken along the AC surface, the angular cylinder ADC slides along the AC surface, and the material is forwardly conveyed in the trough accompanied by the flipping motion, thereby reducing the conveying efficiency and increasing energy consumption of the pipe chain conveyor, so that the driving power is increased. Therefore, angular cylinder ADC was studied, and it was assumed that [6]:

(1) In this paper, the ideal granular medium calculation model is selected;

(2) Any point on plane $\mathrm{AC}$ is in extreme equilibrium;

(3) The angular cylinder ADC is treated as an absolute rigid body. The surface force and volume force acting on it can be replaced by their combined force;

(4) In addition, the materials are regarded as not sticky at all;

(5) The research unit is considered as being in the extreme equilibrium state, that is, it is in the initial instantaneous state of material sliding and scraper displacement. Thus it is assumed that the reaction force acting on the ADC of the sliding angular cylinder from the direction of the scraper and the rest of the $\mathrm{AA}_{1} \mathrm{MC}$ portion of the relatively stationary bulk material has an angle of $\phi_{W}$ with the corresponding normal direction of the plane(material friction angle) and $\phi$ (material internal friction angle). In addition, since the study is on the initial moment of the failure process of the element, the equilibrium condition can be applied to the original undeformed element. Moreover, the problem is still considered as a plane problem. 


\subsection{Passive Total Reaction $F_{s}$ on Glide Plane AC}

Force analysis diagram of angular cylinder ADC, as shown in Figure 3., the coordinate system is established with point $\mathrm{D}$ as the origin of coordinates. The forces acting on the angular cylinder are: angular cylinder ADC bulk material gravity $F_{g}$, scraper force $F_{X}$, full reaction $F_{s}$ on slip plane AC, friction $F_{w}$ of the trough on bulk material.

Take a triangular element at point $\mathrm{E}$, as shown in Figure 4. By analyzing the stress of this element, it can be concluded that $F_{s}^{\prime}$ on surface $\mathrm{AC}$ is:

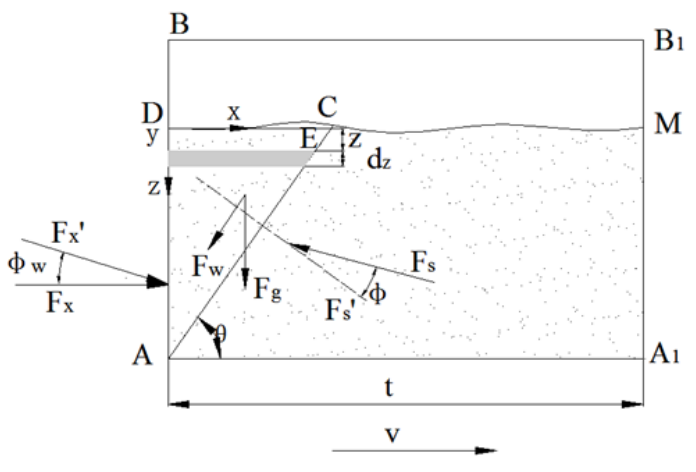

Figure 3. Force analysis of slipping body

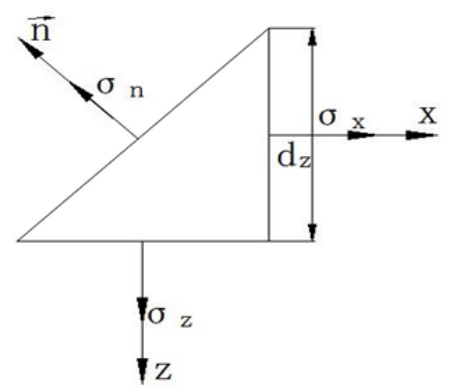

Figure 4. The inclined plane unit

$$
\begin{aligned}
& d F_{\mathrm{s}}^{\prime}=S_{1} z \gamma \cos \theta \sqrt{n_{\text {max }}^{2}+\tan ^{2} \theta}= \\
& 2 \pi \sqrt{\frac{D^{2}}{4}-\left(h-\frac{D}{2}-z\right)^{2}} z \gamma \operatorname{ctg} \theta \sqrt{n_{\text {max }}^{2}+\tan ^{2} \theta} \cdot d z
\end{aligned}
$$

namely

$$
\begin{aligned}
F_{\mathrm{S}}^{\prime} & =\int_{0}^{h} d F_{\mathrm{S}}^{\prime}=\int_{0}^{h} 2 \pi \sqrt{\frac{D^{2}}{4}-\left(h-\frac{D}{2}-z\right)^{2}} \cdot z \gamma \mathrm{ctg} \theta \sqrt{n_{\max }^{2}+\tan ^{2}} \theta \cdot d z \\
& =2 \pi \gamma \mathrm{ctg} \theta \sqrt{n_{\max }^{2}+\tan ^{2} \theta} \\
& {\left[\left(h-\frac{D}{2}\right)\left(\frac{\pi D^{2}}{16}+\frac{D^{2}}{8} \arcsin \frac{2 h-D}{D}+\frac{2 h-D}{4} \sqrt{h D-h^{2}}\right)+\frac{1}{3}\left(h D-h^{2}\right)^{\frac{3}{2}}\right] }
\end{aligned}
$$

So, the passive total reaction on AC surface $F_{s}$ is

$$
\begin{aligned}
F_{\mathrm{S}} & =\frac{F_{\mathrm{S}}^{\prime}}{\cos \varphi} \\
& =\frac{2 \pi \gamma \mathrm{ctg} \theta \sqrt{n_{\max }^{2}+\tan ^{2} \theta}}{\cos \varphi} . \\
& {\left[\left(h-\frac{D}{2}\right)\left(\begin{array}{l}
\left.\frac{\pi D^{2}}{16}+\frac{D^{2}}{8} \arcsin \frac{2 h-D}{D}\right)+\frac{1}{3}\left(h D-h^{2}\right)^{\frac{3}{2}} \\
+\frac{2 h-D}{4} \sqrt{h D-h^{2}}
\end{array}\right)(5)\right.}
\end{aligned}
$$

\subsection{Scraper Force $F_{x}$}

The whole reaction

$$
F_{x}^{\prime}=\frac{F_{x}}{\cos \varphi w}
$$

\subsection{Gravity $F_{g}$ of Angular Cylinder ADC}

$$
\begin{aligned}
& F g=\frac{1}{2} S 1^{\gamma h \operatorname{ctg} \theta=} \\
& \frac{\pi \gamma h}{\sin \theta} \operatorname{ctg} \theta \cdot\left(\frac{\pi_{D}^{2}}{16}+\frac{D}{8} \arcsin \frac{2 h-D}{D}-\frac{2 h D}{4} \sqrt{h D-^{2}{ }^{2}}\right)
\end{aligned}
$$

\subsection{Friction Force $F_{w}$ of ADC of Angular Cylinder of Trough}

It is assumed that the acting direction of $F_{w}$ is opposite to the sliding direction of the platform cylinder along the AC surface, and the minimum lateral pressure shall prevail for calculation.

$$
\begin{aligned}
F_{\mathrm{w}} & =\int_{0}^{h} C \cdot \operatorname{ctg} \theta z \gamma \text { min } \\
& =\frac{D \pi}{360} \operatorname{ctg} \theta \gamma f \min _{\min } \cdot\left\{\frac{(2 h-D)^{2}}{4} \arccos \frac{8 h^{2}-8 h D+D^{2}}{D^{2}}-\left(h-\frac{D}{2}\right) \sqrt{D^{2}-4\left(h-\frac{D}{2}\right)^{2}}\right. \\
& \left.+\frac{1}{16}\left[-\left(8 h^{2}-8 h D+D^{2}\right) \arccos \frac{8 h^{2}-8 h D+D^{2}}{D^{2}}+\sqrt{D^{4}-8 h^{2}+8 h D-D^{2}}\right]\right\}
\end{aligned}
$$

As shown in Figure 3., according to the limit equilibrium condition

$$
\begin{aligned}
& \sum x=0 \\
& F_{X}{ }^{-}{ }_{F s} \cos \left(90^{\circ}-\theta-\phi\right)-{ }_{F}{ }_{w} \cos \theta=0
\end{aligned}
$$

$\sum z=0$

${ }_{F x}{ }^{\prime} \sin \phi_{w}{ }^{+} F_{g}{ }^{+}{ }_{F}{ }_{w} \sin \theta-{ }_{F s} \sin \left(90^{\circ}-\theta-\phi\right)=0$

Substitute equations (6), (7) and (8) into equations (9) and (10) to get 


$$
\begin{gathered}
F_{X}-\frac{1}{3} \gamma n_{w}{ }^{3} \cos \theta_{s} \sin (\theta+\phi) \min \\
F_{X}{ }^{\tan } \phi_{w}+\frac{1}{2} D_{h}^{2} \gamma \operatorname{ctg} \theta+\frac{1}{3} \gamma n 3_{s} \cos (\theta+\phi)_{\min }
\end{gathered}
$$

Equation (11) is divided by equation (12)

$$
\begin{aligned}
& F_{X}=\frac{1}{\operatorname{ctg}(\theta+\phi)-f} \\
& \left\{\begin{array}{c}
31 \\
\left.h \begin{array}{l}
3 \\
\frac{1}{3}
\end{array} \operatorname{ctg} \theta[\cos \theta \bullet \operatorname{ctg}(\theta+\phi)+1]+\frac{1}{2} D_{h}{ }^{2} \gamma \operatorname{ctg} \theta\right\}
\end{array}\right.
\end{aligned}
$$

Make

$$
\begin{aligned}
A_{X}= & \frac{\frac{1}{3} \gamma n f \operatorname{ctg} \theta[\cos \theta \cdot \operatorname{ctg}(\theta+\phi)+1]}{\operatorname{ctg}(\theta+\phi)-f} \\
B_{X}= & \frac{\frac{1}{2} D \gamma \operatorname{ctg} \theta}{\operatorname{ctg}(\theta+\phi)-f}
\end{aligned}
$$

Then

$$
F x=A_{x} h^{3}+{ }_{B x} h^{2}
$$

Since $F_{x}$ is obtained in the limit equilibrium state, it represents the maximum interaction between the scraper and the material during the conveying process of the horizontal pipe chain conveyor. This is also the maximum value of the interaction between the static forces during actual working between the material and scraper.

\section{Propagation Area of Scraper Force and Limit Pitch of Scraper}

As shown in Figure 5., during the material conveying process, the force analysis diagram of the material in a pitch unit abcd is analyzed by taking the average force of the $z=\frac{h}{2}$ position during the research.

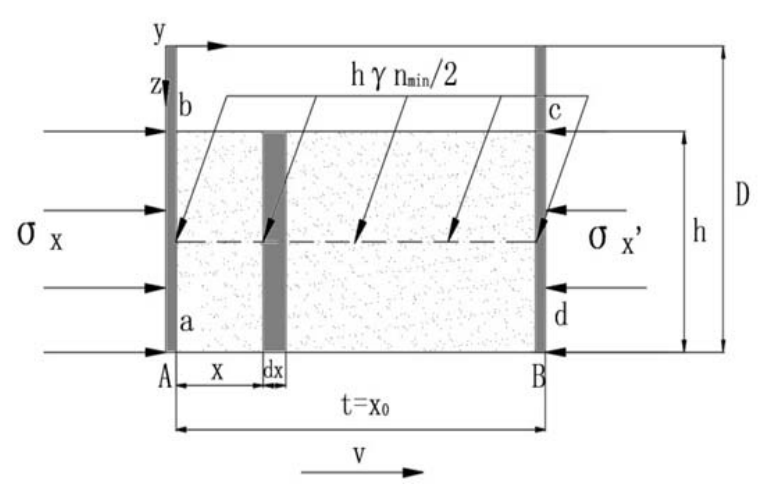

Figure 5. Force analysis diagram of a pitch of material unit
The force of the scraper B on the abcd material

$$
\sigma_{X}^{\prime}=\frac{1}{2} h \gamma_{n \min }
$$

Maximum lateral pressure in the abcd material

$$
\sigma_{X}=\frac{1}{2} h \gamma_{n \max }
$$

According to the mechanics of granular materials:

(1) When $\sigma_{x}^{\prime} \leq \sigma_{x}^{\prime \prime}$, it spreads only in the direction of its action, as shown in Figure 5.

(2) When $\sigma_{x}^{\prime}>\sigma_{x}^{\prime \prime}$, it not only spreads in the direction of its action, but also spreads horizontally, as shown in Figure 6.

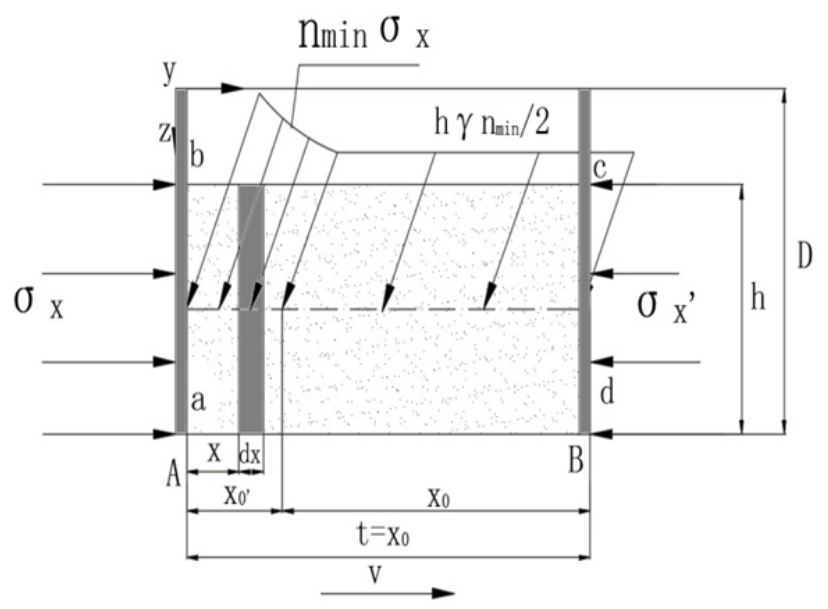

Figure 6. Schematic diagram of the $\sigma_{x}$ lateral spread

\section{1. $\sigma_{x}^{\prime} \leq \sigma_{x}^{\prime \prime}$ situation [7]}

As shown in Figure 5., the unit body selected is shown in Figure 7., and the equilibrium differential equation is

$$
\sigma_{S_{S}}-h \gamma f_{C_{\text {siden } \min d_{X}}-h \gamma f_{C_{\text {base }} d_{X}}-\left(\sigma-{ }_{\sigma_{\sigma}}\right)_{S_{S}}=0}
$$

Namely

$$
d_{X}=\frac{S_{s}}{h \lambda f_{C_{\text {siden } \min }}-h \gamma f_{\text {Cbase }}} d_{\sigma}
$$

In the formula, $\mathrm{C}$ side - perimeter of the trough side wall in contact with the material, $\mathrm{m}$, calculated according to formula (21);

C bottom-perimeter of the bottom of the trough in contact with the material, $\mathrm{m}$, calculated according to formula (22);

$\mathrm{S}_{\mathrm{S}}$ - the area of the scraper in contact with the material, $\mathrm{m}^{2}$, calculated according to equation (23). 


$$
\begin{gathered}
C_{\text {side }}=\frac{1}{2} \pi D-D \arcsin \frac{2 \sqrt{h(D-h)}}{D} \\
C \text { base }=D \arcsin \frac{2 \sqrt{h(D-h)}}{D} \\
S_{S}=\frac{D^{2}}{4}\left(\pi-\arcsin \frac{2 \sqrt{h(D-h)}}{D}\right)+\left(h-\frac{D}{2}\right) \sqrt{h(D-h)}
\end{gathered}
$$

Assuming the propagation distance of $\sigma_{x}$ is $x_{0}$, then the integral is determined in $x \in\left[0, x_{0}\right]$ and

$$
\begin{gathered}
\sigma \in\left[\sigma_{x}^{\prime}, \sigma_{x}\right] . \\
\int_{0}^{x 0} d x=\int_{\sigma_{x}}^{\sigma_{1}^{x}} \frac{S_{s}}{f h \gamma_{n_{\min } C_{\text {side }}+h \gamma f_{C \text { base }}}}
\end{gathered}
$$

Then

$$
x_{0}=\frac{S_{s}}{f h \gamma_{n_{\min } C_{\text {side }}+h \gamma f_{\text {Cbase }}}}\left(\sigma_{x}-\sigma_{x}\right)
$$

In the formula, $\sigma_{x}$ is determined by the formulas (2) and (13) [8].

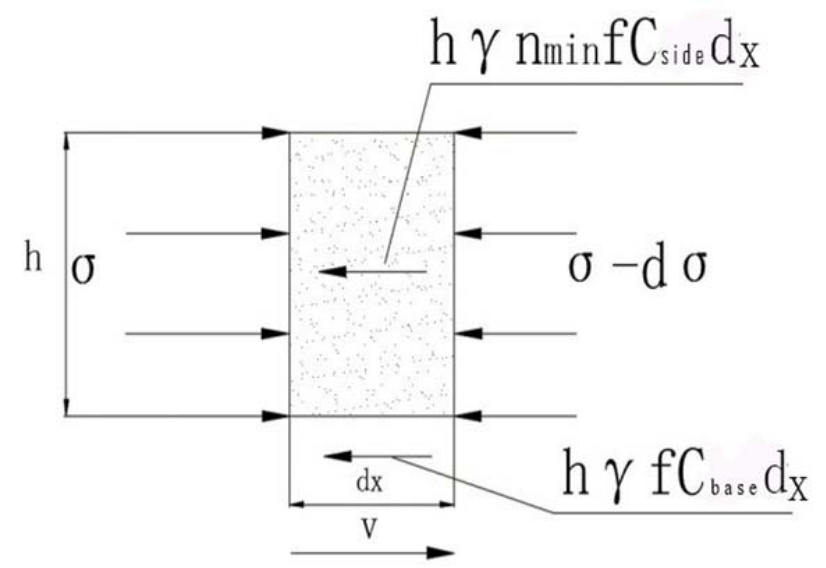

Figure 7. The unit body of Figure 5. Corresponded

\section{2. $\sigma_{x}>\sigma_{x}^{\prime \prime}$ situation [9]}

Corresponding to Figure 6., the unit body selected is shown in Figure 8. At this time, the range of action of $\sigma_{x}$ can be divided into two sections $x_{0}$ and $x_{0}{ }^{\prime}$. In the interval of $x_{0}, \sigma_{x}$ only propagates along its direction of action; in the $x_{0}^{\prime}$ interval, $\sigma_{x}$ not only propagates along its direction of action, but also spreads laterally to it. Set the side pressure coefficient of its propagation be $n_{\min }$, then the side pressure of $\sigma_{x}$ to the lateral propagation is $\sigma_{x} n_{\min }$, and there is equilibrium differential equation:

$\sigma_{S_{S}}-2 \sigma f n_{\min } C_{\text {side }_{X}}-\sigma f n_{\min } C_{\text {base }} d_{X}-\left(\sigma-d_{\sigma}\right)_{S_{S}}=0$

Namely

$$
d_{X}=\frac{S_{S}}{2 \sigma f n_{\min } S_{\text {side }}+\sigma f n_{\text {min }} C_{\text {base }}} d \sigma
$$

The definite integral of $x \in\left[0, x_{0}^{\prime}\right]$ and $\sigma \in\left[\sigma_{x}^{\prime \prime}, \sigma_{x}\right]$

$\int_{0}^{x_{0}} d x=\frac{\mathrm{S}_{\mathrm{s}}}{2 \mathrm{fn}_{\text {min }} C_{\text {side }}+\mathrm{fn}_{\text {min }} C_{\text {base }}} \int_{\sigma_{x}^{\prime}}^{\sigma_{x}} \frac{d \sigma}{\sigma}$

$X_{0}^{\prime}=\frac{\mathrm{S}_{\mathrm{s}}}{2 \mathrm{fn}_{\text {min }} C_{\text {side }}+\mathrm{fn}_{\text {min }} C_{\text {base }}} \ln \left(\sigma_{x}-\sigma_{x}{ }^{\prime \prime}\right.$

This is the length of the region where $\sigma_{x}$ is propagating laterally, so the action range of $\sigma_{x}$ is

$$
\begin{aligned}
& x 0+x^{\prime} 0=\frac{\mathrm{S}_{\mathrm{S}}}{\mathrm{fh} \gamma \mathrm{n}_{\text {min }} C_{\text {side }}+\mathrm{h} \gamma \mathrm{f} C_{\text {base }}}\left(\sigma_{X}-\sigma_{X}^{\prime}\right)+x_{0}^{\prime} \\
& =\frac{\mathrm{S}_{\mathrm{s}}}{2 \mathrm{fn}_{\text {min }} C_{\text {side }}+\mathrm{fn}_{\text {min }} C_{\text {base }}} \ln \left(\sigma_{X}-\sigma_{X}^{\prime \prime}\right)
\end{aligned}
$$

Substitute equation (21), (22) and (23) into equation (29) to get the final expression of the action range of $\sigma_{x}$

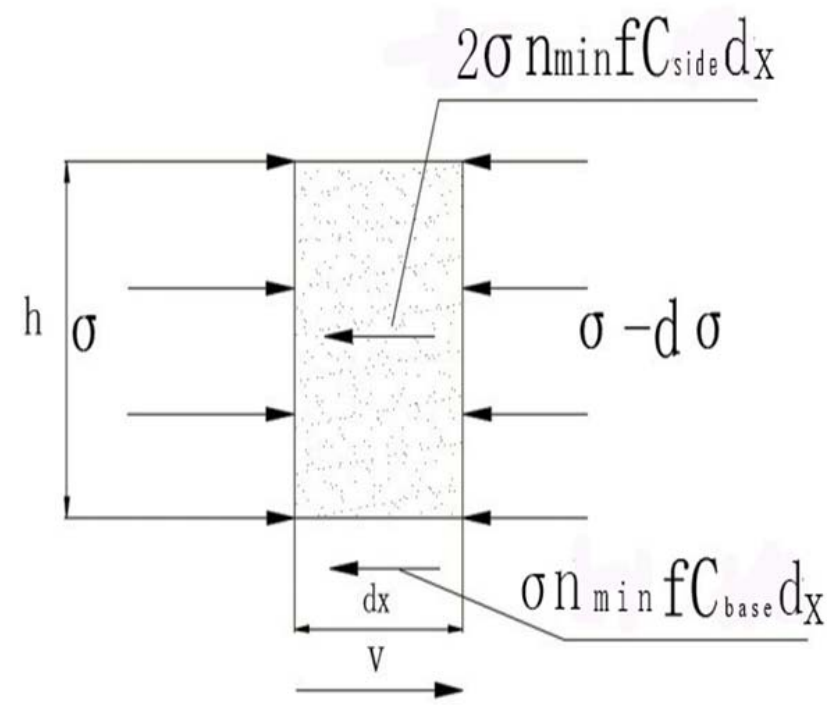

Figure 8. The unit body of Figure 6. corresponded 


$$
\begin{aligned}
& x_{0}+x_{0}^{\prime}=\frac{\left[\frac{D^{2}}{4}\left(\pi-\arcsin \frac{2 \sqrt{h(D-h)}}{D}\right)+\left(h-\frac{D}{2}\right) \sqrt{h(D-h)}\right]\left(\sigma_{\mathrm{x}}-\sigma_{\mathrm{x}}^{\prime}\right)}{\frac{1}{2} \pi D / h n_{\text {min }_{\text {in }}}-h \gamma f \text { arcsin } \frac{2 \sqrt{h(D-h)}}{D}\left(n_{\text {min }}-1\right)}+ \\
& {\left[\frac{D^{2}}{4}\left(\pi-\arcsin \frac{2 \sqrt{h(D-h)}}{D}\right)+\left(h-\frac{D}{2}\right) \sqrt{h(D-h)}\right] \ln \left(\sigma_{x}-\sigma_{x}^{\prime \prime}\right)} \\
& f n_{\min } \pi D-f n_{\min } D \arcsin \frac{2 \sqrt{h(D-h)}}{D}
\end{aligned}
$$

Obviously, if the scraper is to transport materials, there must be $\sigma_{x}>\sigma_{x}{ }^{\prime \prime}$, Therefore, in the disc pipe scraper conveyor, the propagation range of the force $F_{X}$ acting on the material by the scraper is limited by the formula (30).

Assuming $t_{1}$ is a limit value of the scraper pitch t, then

$$
t_{1}=x_{0}+x_{0}^{\prime}
$$

\section{Conclusion}

Based on the introduction of the structural composition and working principle of the pipe chain conveyor, this paper applies the theoretical theory, the theory of bulk mechanics and the basic theory of soil mechanics retaining wall, and analyzes the conveying material for the special round pipe trough of the pipe chain conveyor. The interaction between the scraper and the material at the limit equilibrium state is as follows:

(1) According to the limit equilibrium condition of the bulk material, the maximum interaction force between the scraper and the material during the process of conveying material in the horizontal pipe chain conveyor when the material is in the limit equilibrium state is derived.

(2) By analyzing the propagation area of the scraper force of the pipe chain conveyor, the limit pitch of the scraper when the material is in the limit equilibrium state is given, that is, the pitch of the scrapers should meet $t_{1}=x_{0}+x_{0}^{\prime}$ when designing the pipe chain conveyor.

The research of the thesis provides a theoretical basis for the rational design and calculation of the pipe chain conveyor, and is of great significance to promote the development of the domestic pipe chain conveyor.

\section{References}

[1]. Yao Y. P. (2015). Research on discrete element simulation and multi-attribute evaluation of disc pipe scraper conveyor, M.A.Thesis. Shanxi:Yaiyuan University of Science and Technology.

[2]. Yao Y. P, Zhai Z. M, Meng W. J. (2013). Mechanical analysis of bulk material in vertical section of pipeline scraper conveyor. Hoisting and Transport Machinery, (09), 34-37.

[3]. LI W. (2012). Research on microstructure mechanism of basic mechanical properties of cohesive soil, M.A.Thesis. Shanghai: Tongji University.

[4]. Han G, Wan X. J. (2006). Mechanism analysis of increasing inclination angle of deep groove belt conveyor. Hoisting and Transport Machinery, (5), 1517.

[5]. Huang S. Y. (2000). Dispersion mechanics. Beijing: Mechanical Industry Press.

[6]. Meng W. J, Huang S. Y, Wang Y. (1992). Experimental research on horizontal large-capacity buried scraper conveyor. Journal of Taiyuan University of Science and Technology, (4), 16-29.

[7]. Furmanik, K., \& Kasza, P. (2010). Additional Resistances Of Movement Of A Scraper Pipe Conveyor. Tribologia(Poland), 41(3), 49-62.

[8]. Krause, F., \& Katterfeld, A. (2004). Functional analysis of tube chain conveyors. Particle \& Particle Systems Characterization: Measurement and Description of Particle Properties and Behavior in Powders and Other Disperse Systems, 21(4), 348-355.

[9]. Schmolke, S., \& Katterfeld, A. (2001). Measurement Signals of the Chain Tractive Force from a Closed Pipe Circuit. In Conference Proceedings MAT, Messegesellschaft Nurnberg. 